\title{
UTILIZAÇÃO DE RESÍDUOS DA MADEIRA DE CANDEIA (Eremanthus erythropappus (DC.) Macleish) NA PRODUÇÃO DE PAINÉIS AGLOMERADOS COM ADIÇÃO DE PET
}

\author{
UTILIZATION OF CANDEIA (Eremanthus erythropappus (DC.) Macleish) WOOD RESIDUES IN THE \\ PRODUCTION OF PARTICLEBOAD WITH ADDITION OF PET
}

Rosimeire Cavalcante dos Santos ${ }^{1}$ Lourival Marin Mendes ${ }^{2}$ Angélica de Cássia Oliveira Carneiro ${ }^{3}$ Fábio Akira Mori ${ }^{4}$ Renato Vinícius Oliveira Castro ${ }^{5}$ Rafael Farinassi Mendes ${ }^{6}$

\begin{abstract}
RESUMO
Este trabalho objetivou analisar, por meio das propriedades físicas e mecânicas, a viabilidade da produção de painéis a partir da incorporação do resíduo da madeira de candeia (Eremanthus erythropappus) e a influência das diferentes porcentagens de plástico tipo PET (polietileno tereftalato), como também da presença e ausência de parafina sobre as propriedades dos painéis aglomerados. Foram utilizados resíduos da madeira de candeia, após a extração do óleo, em associação à madeira de eucalipto, na proporção de 25:75, e adesivo uréia-formaldeído (12\%) para a produção dos painéis; além da incorporação de PET na forma de partículas originadas de envases de refrigerantes em três porcentagens de incorporação $(0 \%$, $25 \%$ e $50 \%$ ) em tratamentos na presença (1\%) e ausência de emulsão de parafina. O ciclo de prensagem dos painéis ocorreu sob aquecimento elétrico a temperatura de $160 \mathrm{oC}$; pressão de $0,4 \mathrm{MPa}$ e tempo de 8 minutos. $\mathrm{O}$ experimento foi instalado em um delineamento inteiramente casualizado com três repetições. As propriedades avaliadas, segundo as normas DIN (1971), ASTM D 1037-93 (1995) e CS 236-66 (1968), foram: ligação interna; flexão estática (módulo de elasticidade - MOE e módulo de ruptura - MOR); compressão paralela à superfície das chapas; absorção de água e inchamento em espessura, após 2 e 24 horas de imersão. As propriedades mecânicas dos painéis foram reduzidas com o aumento dos níveis de incorporações de PET; a adição de parafina não promoveu, de modo geral, ganhos de resistência e redução de higroscopicidade dos painéis madeira-plástico; é viável a utilização do resíduo da madeira de candeia, em associação à madeira de eucalipto, na confecção dos painéis madeira-plástico, pois as propriedades atenderam às exigências mínimas das normas, exceto para o teste de flexão estática.
\end{abstract}

Palavras-chave: resíduo de madeira; uréia-formaldeído; painéis aglomerados.

\begin{abstract}
This work aimed to evaluate, through the physical and mechanical properties, the panels production viability with inclusion of candeia (Eremanthus erythropappus) wood residues and the influence of different percentages of PET (polyethylene terephthalate), as well as the presence and absence of paraffin on the properties of particleboard. There were used candeia wood residues, after oil extraction, in association with eucalypt wood in the proportion of 25:75 and urea-formaldehyde adhesive (12\%) for panels production; besides the PET incorporation in particle form, which were originated from soft drink bottles and included in three percentages $(0 \%, 25 \%$ e $50 \%)$ in treatments in the presence $(1 \%)$ and absence of paraffin emulsion.

1. Engenheira Florestal, $\mathrm{Dr}^{\mathrm{a}}$, Pesquisadora do Departamento de Ciências Florestais, Universidade Federal de Viçosa, CEP 36570-000, Viçosa (MG). meire_caico@yahoo.com.br

2. Engenheiro Florestal Dr., Professor do Departamento de Ciências Florestais - UFLA, Caixa Postal 3037, CEP 37200-000, Lavras (MG). lourival@ufla.br

3. Engenheira Florestal, Dr $^{\mathrm{a}}$, Professora do Departamento de Ciências Florestais, Universidade Federal de Viçosa, CEP 36570-000, Viçosa (MG). cassiacarneiro@ufv.br

4. Engenheiro florestal, Dr., Professor do Departamento de Ciências Florestais, Universidade Federal de Viçosa, CEP 36570-000, Viçosa (MG). morif@ufla.br

5. Engenheiro Florestal, Mestrando em Ciências Florestais, Universidade Federal de Viçosa, CEP 36570-000, Viçosa (MG).castrorvo@ymail.com

6. Engenheiro Florestal, Doutorando em Ciência e Tecnologia da Madeira, Universidade Federal de Lavras, CEP 37200-000, Lavras (MG). rafaelfarinassi@gmail.com

Recebido para publicação em 3/04/2008 e aceito em 2/09/2010
\end{abstract}


The panels pressing cycle occurred under electric heating at $160^{\circ} \mathrm{C}, 0.4 \mathrm{MPa}$ of pressure, during 8 minutes. The experimental design was entirely randomized with three repetitions. The properties evaluated, according to DIN (1971), ASTM D 1037-93 (1995) and CS 236-66 (1968) standards, were: internal bonding; static bending (modulus of elasticity - MOE and rupture - MOR); compression parallel to the panel surface; water absorption and thickness swelling, after 2 and 24 hours water soaking. The panel mechanical properties decreased with increasing in PET level; in general, paraffin addition did not improve the wood/plastic panels resistance and higroscopicity; the utilization of candeia wood residues is viable, in association with eucalypt wood, for the wood/plastic panel production, since the properties attended the minimum demands of the standards, except static bending.

Keywords: wood residue; urea-formaldehyde; particleboard.

\section{INTRODUÇÃO}

No setor madeireiro, a grande quantidade de resíduos gerados sempre foi motivo para preocupações. Dentre esses, o resíduo gerado a partir do processo de extração do óleo da madeira de candeia merece especial atenção, pois $o$ volume gerado pelas fábricas que extraem o óleo bruto é superior àquele utilizado como forma de energia durante o processo de extração, o que gera problemas com armazenamento e contaminação do ambiente. Atualmente, cada vez mais os resíduos vêm despertando o interesse de pesquisadores e empresários, principalmente para verificar as possibilidades de reutilização desses materiais.

Uma das alternativas para os resíduos gerados pelas fábricas produtoras do óleo de candeia é a sua utilização na produção de painéis particulados. Os painéis madeira-plástico são uma variação dos painéis conhecidos como chapas de partículas aglomeradas. Além da madeira, fonte principal de matéria-prima para a produção de chapas de partículas, outros polímeros, principalmente aqueles de composição termoplástica, têm sido testados, fundidos ou em forma de partículas, para a elaboração desses produtos. Durante a fusão desses polímeros, no entanto, o calor afeta negativamente a maioria de suas propriedades. Na tentativa de manter inalteradas suas estruturas, novos tipos de plástico têm sido avaliados, em suas formas fragmentadas, dentre esses, o PET (polietileno tereftalato) é utilizado como elemento constituinte para a elaboração de materiais à base de madeira $\mathrm{e}$ plástico.

O uso de plástico em mistura com a madeira na produção dos compósitos madeira-plástico está crescendo rapidamente. Isso se deve às vantagens desse produto em relação à madeira, como não rachar, não empenar e exigir pouca ou nenhuma manutenção (BRANDT e FRIDLEY, 2003), além de promover aumento da estabilidade dimensional do produto e proteção contra micro-organismos.

Falk et al. (1999) compararam painéis de madeira com painéis de madeira-plástico (polietileno e polipropileno). Os autores afirmam que, em geral, os compósitos de madeira-plástico apresentaram desempenho inferior aos painéis de madeira, entretanto, os resultados foram satisfatórios, levando-se em consideração a norma CS 236-66. De acordo com Harper (1996), o PET é um termoplástico polar, com elevadas estabilidade dimensional e temperatura de fusão, o que o coloca em vantagem, no que se refere à maior estabilidade, pois maiores serão as possibilidades de utilizá-lo em diferentes ambientes.

Vale ressaltar que a tecnologia de produção dos compósitos termoplásticos reforçados com fibras lignocelulósicas utilizando PET requerem temperatura de fusão em torno de $250^{\circ} \mathrm{C}$. No entanto, a utilização dessa faixa de temperatura na produção de painéis aglomerados com inclusão do referido termoplástico pode tornar inviável economicamente esse processo, especialmente quando se pretende aproveitar resíduo madeireiro.

A parafina é usualmente adicionada às chapas de partículas de madeira aglomerada, produzidas sem a incorporação de polímeros, a fim de proporcionar aumento na resistência do painel acabado quanto à absorção de água na forma líquida. Contudo, isso não melhora o desempenho do painel quanto à absorção de vapor d'água. A inclusão de polímeros poderia promover ao compósito melhoria da resistência à absorção de água na forma de vapor.

A candeia (Eremanthus erythropappus) é uma espécie florestal de múltiplos usos, podendo ser utilizada como moirões de cerca de alta durabilidade, além de ser produtora de óleos essenciais. Outras razões para se investir na candeia é que ela é uma espécie nativa e os produtos dela obtidos alcançam preços relativamente altos no mercado.

Assim, para viabilizar a produção de painéis particulados a partir do aproveitamento do resíduo 
da candeia é necessário maior conhecimento sobre a utilização desse resíduo após a extração do óleo, como também sobre a quantidade gerada para atender a esse propósito.

Desse modo, o presente trabalho foi realizado com o objetivo de analisar, por meio das propriedades físicas e mecânicas, a viabilidade da produção de painéis a partir da incorporação do resíduo da madeira de candeia (Eremanthus erythropappus), bem como a influência das diferentes porcentagens do plástico tipo PET (polietileno tereftalato) e da presença e ausência de parafina sobre as propriedades dos painéis aglomerados.

\section{MATERIAL E MÉTODO}

Foram utilizados nesta pesquisa: resíduos da madeira de candeia (Eremanthus erythropappus), após a extração do óleo, procedentes da indústria de óleo de candeia CITROMINAS, localizada no município de Carrancas - MG; madeira de Eucalyptus spp., coletada no Campus da Universidade Federal de Lavras, MG; e polietileno tereftalato (PET), obtido de uma fábrica de materiais recicláveis localizada no município de Bonsucesso, MG, na forma de partículas originadas de envases de refrigerantes, já isentos de impurezas.

Na confecção dos painéis foram utilizados também adesivo ureia-formaldeído com teor de sólidos de 62,37\%, polietileno tereftalato (PET) e emulsão de parafina com teor de sólidos de 59,8\%.

Foram produzidas partículas das madeiras de eucalipto e candeia em moinho martelo, após serem processadas em picador. Essas partículas foram secas em estufa a $103^{\circ} \mathrm{C}$ até atingir o conteúdo de umidade de $3 \%$, base massa seca da madeira.

As partículas de madeira e as de PET foram classificadas em peneiras sobrepostas, sendo utilizadas na confecção dos painéis aquelas retidas em peneira com malha de $1,0 \times 1,0 \mathrm{~mm}$.

A densidade do PET foi determinada medindo-se o volume de água deslocada por uma massa conhecida e a densidade básica média das madeiras de eucalipto e candeia foram determinadas de acordo com Vital (1984).

Todos os painéis foram produzidos de forma a se obter uma densidade final igual a $0,70 \mathrm{~g} / \mathrm{cm}^{3}$ e dimensões de $48 \times 48 \times 1,5 \mathrm{~cm}$. As frações de madeira e plástico foram calculadas em função desses parâmetros. Para o cálculo da massa de madeira, considerou-se umidade de 3\%. As quantidades de adesivo e parafina aplicadas nos painéis foram de $12 \%$ e $1 \%$, respectivamente, com base na massa da mistura empregada. A massa de partículas foi calculada para gerar um painel por vez, cada tratamento correspondendo a três repetições.

As massas das partículas de madeira e do plástico foram pesadas e colocadas no interior de um misturador tipo tambor rotatório, à velocidade de 30 rotações por minuto. $\mathrm{O}$ adesivo e a parafina foram aplicados por meio de uma pistola acionada por compressor de ar, durante um tempo médio de aplicação de 6 minutos. O método consistiu em adicionar primeiro o adesivo e, logo após, a parafina.

$\mathrm{O}$ adesivo foi colocado em recipiente graduado e descia até a pistola por gravidade; a parafina foi colocada em um recipiente no interior do tambor. A mistura de partículas de madeira e plástico, depois de aspergidas com o adesivo e a parafina, foi levada para a seção formadora do colchão.

O colchão foi formado, manualmente, depositando-se a mistura de partículas sobre uma chapa de alumínio de 2,0 $\mathrm{mm}$ de espessura, colocada sob uma caixa de madeira sem fundo, com dimensões internas de $48 \times 48 \mathrm{~cm}$. As partículas foram espalhadas de forma aleatória e levadas a uma pré-prensagem manual, sob pressão média de $0,1 \mathrm{MPa}$, durante 3 minutos.

Após a pré-prensagem manual, o colchão de partículas foi levado à prensa hidráulica com a mesma chapa de alumínio recebida na préprensagem e outra semelhante na parte superior do colchão. Para controlar a espessura final do painel em $1,5 \mathrm{~cm}$ e evitar uma possível interferência na sua densidade, foram colocadas barras nas laterais do colchão antes do mesmo ser submetido à pressão em prensa hidráulica automática, com aquecimento elétrico. $\mathrm{O}$ ciclo de prensagem obedeceu às seguintes condições: temperatura de $160^{\circ} \mathrm{C}$; pressão de $0,4 \mathrm{MPa}$ e tempo de 8 minutos.

A retirada dos corpos-de-prova foi realizada após acondicionamento dos painéis na câmara de climatização à temperatura de $20 \pm 2^{\circ} \mathrm{C}$ e umidade relativa de $65 \pm 3 \%$ até atingirem peso constante. Inicialmente, os painéis foram esquadrejados, retirando-se cerca de $2 \mathrm{~cm}$ das bordas e, em seguida, adotaram-se especificações das normas DIN (1971), com as devidas modificações. Para a avaliação das propriedades físico-mecânicas dos painéis, foram utilizadas referências das normas ASTM D 1037 (1993) e CS 236-66. 
Foram retirados os corpos-de-prova para os seguintes ensaios físicos e mecânicos: ligação interna (LI), flexão estática (módulo de elasticidade - MOE e módulo de ruptura - MOR), compressão paralela (CP), absorção de água (AA) e inchamento em espessura (IE), após 2 e 24 horas de imersão.

Adotaram-se 6 tratamentos com três repetições, totalizando 18 painéis. $\mathrm{O}$ experimento foi instalado segundo um delineamento inteiramente casualizado com três repetições. Os tratamentos foram arranjados segundo um esquema fatorial $3 \mathrm{x}$ 2 , sendo 3 porcentagens de incorporação de plástico $\operatorname{PET}(0 \%, 25 \%$ e $50 \%)$, na presença e na ausência de parafina.

O modelo estatístico que descreve as observações é dado por:

$$
\mathrm{y}_{i j k}=\mu+s_{i}+p_{j}+s p_{i j}+\varepsilon_{i j k}
$$

Em que: $y_{i j k}$ é o valor da variável dependente na k-ésima repetição que recebeu a i-ésima porcentagem de incorporação de PET no j-ésimo nível do fator parafina, com $\mathrm{k}=1,2,3$; $\mu$ é uma constante inerente a cada observação; s é o efeito da j-ésima porcentagem de incorporação de PET, com $\mathrm{i}=1,2,3 ; \mathrm{p}_{\mathrm{j}}$ é o efeito do j-ésimo nível do fator parafina, $\mathrm{j}=1,2$; $\mathrm{sp}_{\mathrm{ij}}$ é o efeito da interação entre a j-ésima porcentagem de incorporação de PET e o j-ésimo nível do fator parafina; $\square_{\mathrm{ijk}}$ é o erro experimental associado à observação na k-ésima repetição que recebeu a i-ésima porcentagem de incorporação de PET no j-ésimo nível do fator parafina, independente e com distribuição normal com média zero e variância $\sigma_{2}$.

$\mathrm{O}$ delineamento experimental adotado na confecção dos painéis está apresentado na Tabela 1.

TABELA 1: Delineamento experimental empregado na confecção dos painéis.

TABLE 1: Experimental design used in panel manufacture.

\begin{tabular}{|c|c|c|c|c|c|}
\hline \multirow{2}{*}{ 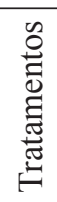 } & \multicolumn{2}{|c|}{$\begin{array}{c}\text { Composição das } \\
\text { partículas de madeira }\end{array}$} & \multirow{2}{*}{$\begin{array}{l}\stackrel{0}{0} \\
\frac{\pi}{0} \\
\frac{\pi}{0} \\
\sum\end{array}$} & \multirow{2}{*}{$\underset{\substack{1 \\
2}}{\stackrel{2}{2}}$} & \multirow{2}{*}{ 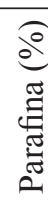 } \\
\hline & $\begin{array}{c}\text { Candeia } \\
(\%)\end{array}$ & $\begin{array}{c}\text { Eucalipto } \\
(\%)\end{array}$ & & & \\
\hline $\mathrm{T} 1$ & 25 & 75 & 100 & 0 & 1 \\
\hline $\mathrm{T} 2$ & 25 & 75 & 100 & 0 & 0 \\
\hline $\mathrm{T} 3$ & 25 & 50 & 75 & 25 & 1 \\
\hline $\mathrm{T} 4$ & 25 & 50 & 75 & 25 & 0 \\
\hline T5 & 25 & 25 & 50 & 50 & 1 \\
\hline T6 & 25 & 25 & 50 & 50 & 0 \\
\hline
\end{tabular}

\section{RESULTADOS E DISCUSSÃO}

As densidades médias observadas do PET e das madeiras de eucalipto e candeia foram iguais a $1,13 \mathrm{~g} / \mathrm{cm}^{3}, 0,57 \mathrm{~g} / \mathrm{cm}^{3}$ e $0,58 \mathrm{~g} / \mathrm{cm}^{3}$, respectivamente.

\section{Propriedades físicas}

Os resultados apresentados na análise de variância indicam que, para a variável absorção de água em 2 horas, houve efeito significativo das fontes de variação isoladamente, ou seja, a porcentagem de incorporação de PET e parafina (I x P) atua de maneira independente nessa variável. Para a absorção de água em 24 horas, verificouse que a interação foi significativa e a absorção de água se comporta de maneira diferenciada nas porcentagens de incorporação de PET, na presença e na ausência de parafina.

Na Figura 1, se observa que existe relação quadrática entre a absorção de água medida em 2 horas e a porcentagem de incorporação de plástico do tipo PET utilizado em associação à madeira. Houve redução na absorção de água em 2 horas com o aumento da porcentagem de PET associado, sendo o mínimo de absorção de água conseguido para, aproximadamente, $25 \%$ de plástico.

Os valores médios de absorção de água (AA) após 2 e 24 horas de imersão, em função das

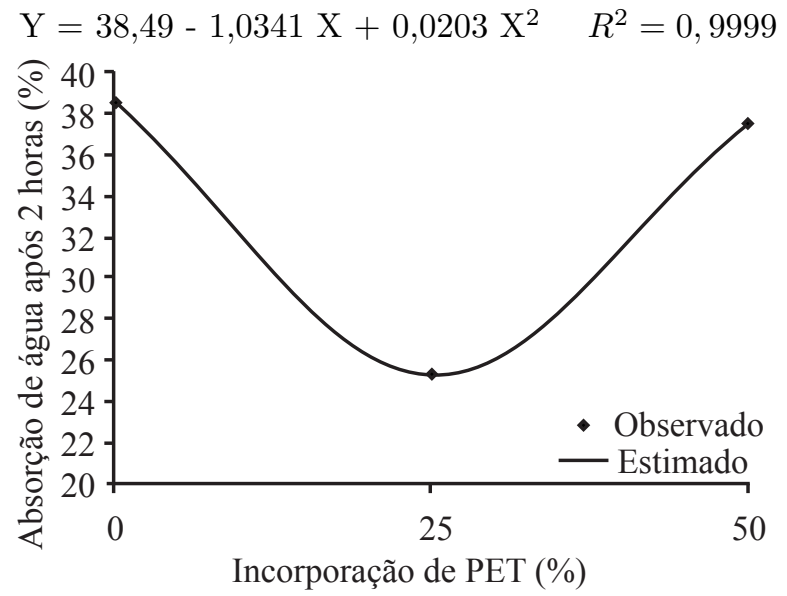

FIGURA 1: Valores médios de absorção de água após 2 horas de imersão, em função das porcentagens de incorporação de PET em associação à madeira.

FIGURE 1: Average values of water absorption after 2 hours of immersion, as a function of PET percentage combined with wood. 
porcentagens de incorporação de PET e ausência ou presença de parafina, estão apresentados nas Tabelas 2 e 3 , respectivamente.

No que se refere à presença ou não de parafina na confecção de painéis, a absorção de água após 2 horas de imersão foi significativamente inferior quando os painéis foram confeccionados aplicando-se a parafina.

Maciel (2001), estudando chapas de partículas aglomeradas em mistura com dois tipos de plástico, um deles PET, em dois níveis de incorporação, e adição de solução de poliestireno em tolueno encontrou, para todos os tratamentos, redução significativa na absorção de água pelos painéis. Esse autor não utilizou parafina na confecção dos painéis. Vale ressaltar, conforme observado na Tabela 2, que a adição de parafina também reduziu a absorção, o que pode ter contribuído para o aumento da estabilidade dimensional dos painéis.

Observa-se que, para a incorporação de 50\% de PET, ocorreu acréscimo na absorção de água pelos painéis, o que não era esperado, visto que o PET é hidrofóbico. Provavelmente, essa maior absorção se deve à menor taxa de compressão aplicada a esses painéis em função da maior densidade do PET $\left(1,14 \mathrm{~g} / \mathrm{cm}^{3}\right)$, o que pode ter dificultado a compactação das partículas de madeira, deixando as mesmas disponíveis para maior absorção, devido à maior área superficial.

Os resultados apresentados para a análise de desdobramento indicaram que o efeito dos níveis de substituição foi significativo para a absorção de água em 24 horas apenas para os painéis confeccionados na ausência de parafina.

Nota-se, na Figura 2, uma relação linear decrescente entre a absorção de água em 24 horas e a porcentagem de incorporação de PET nos painéis madeira-plástico, na ausência de parafina.

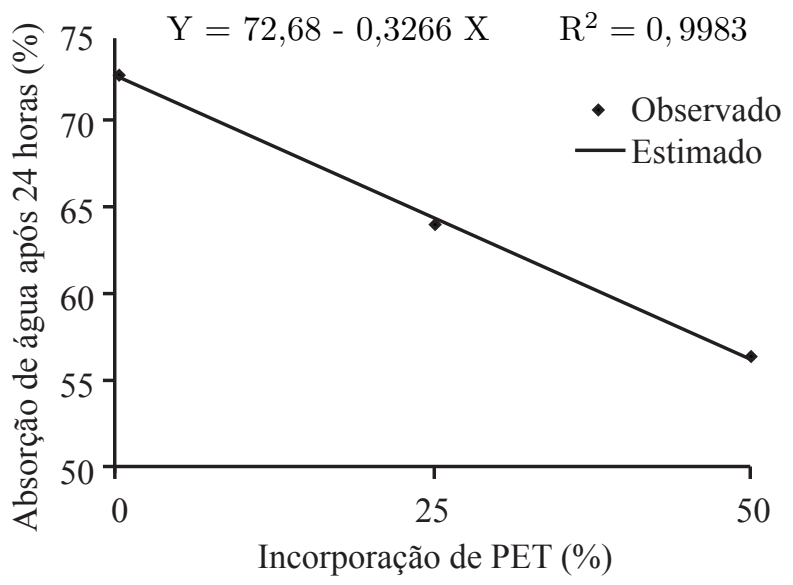

FIGURA 2: Valores médios de absorção de água após 24 horas de imersão, em função das porcentagens de incorporação de PET, na ausência de parafina.

FIGURE 2: Average values of water absorption after 24 hours of immersion, as a function of PET percentage combined with wood in paraffin absence.

TABELA 2: Porcentagem de absorção de água após 2 horas de imersão, em função da porcentagem de incorporação de plástico tipo PET, na presença/ausência de parafina (erro padrão da média).

TABLE 2: Water absorption percentage after immersion for 2 hours as a function of the percentage of PET incorporation in the paraffin presence/absence (average standard error).

\begin{tabular}{ccccc}
\hline \multirow{2}{*}{ Parafina } & \multicolumn{3}{c}{ Porcentagem de incorporação de PET } & \multirow{2}{*}{ Médias $(\%)$} \\
\cline { 2 - 4 } & $0 \%$ & $25 \%$ & $50 \%$ & \\
\hline Presença & $27,46(4,34)$ & $17,17(4,34)$ & $33,19(4,34)$ & $25,94(2,51) \mathrm{b}$ \\
Ausência & $49,51(4,34)$ & $33,46(4,34)$ & $41,81(4,34)$ & $41,59(2,51) \mathrm{a}$ \\
\hline Médias (\%) & $38,49(3,07)$ & $25,31(3,07)$ & $37,50(3,07)$ & \\
\hline
\end{tabular}

Médias seguidas de mesma letra minúscula na coluna não diferem entre si, pelo teste t de Student, a 5\% de significância.

TABELA 3: Porcentagem de absorção de água após 24 horas de imersão em função da porcentagem de incorporação de plástico tipo PET na presença/ausência de parafina (erro padrão da média).

TABLE 3: Average values of water absorption after 24 hours of immersion, as a function of PET percentage combined with wood in paraffin presence/absence.

\begin{tabular}{ccccc}
\hline \multirow{2}{*}{ Parafina } & \multicolumn{3}{c}{ Porcentagem de incorporação de PET } & \multirow{2}{*}{ Médias $(\%)$} \\
\cline { 2 - 4 } & $0 \%$ & $25 \%$ & $50 \%$ & $54,49(1,35) \mathrm{b}$ \\
Presença & $54,34(2,34) \mathrm{b}$ & $54,80(2,34) \mathrm{b}$ & $54,32(2,34) \mathrm{a}$ & $540,51(1,35) \mathrm{a}$ \\
\hline Ausência & $72,87(2,34) \mathrm{a}$ & $64,12(2,34) \mathrm{a}$ & $56,54(2,34) \mathrm{a}$ & $64,51(1,65)$ \\
\hline Médias $(\%)$ & $63,61(1,65)$ & $59,46(1,65)$ & $55,43(1,65)$ & \\
\hline
\end{tabular}

Médias seguidas de mesma letra minúscula na coluna não diferem entre si, pelo teste t de Student, a 5\% de significância. 
Os valores de absorção de água após $24 \mathrm{~h}$ de imersão apresentados na Tabela 3 mostram que os painéis produzidos na ausência de plástico e aqueles produzidos com $25 \%$ de incorporação de PET absorveram, de forma significativa, menor porcentagem de água quando confeccionados com parafina. Para 50\% de incorporação de PET à madeira, não houve diferença significativa, em função da presença ou não de parafina na confecção dos painéis.

Verifica-se, portanto, um efeito positivo da adição de parafina aos painéis madeiraplástico até a incorporação de $25 \%$ de PET. Acima desse valor o caráter hidrofóbico do PET foi maior, podendo ser dispensado o uso da parafina, o que acarreta economia na produção dos painéis.

No entanto, as menores médias percentuais observadas para absorção de água na presença de parafina reforçam o caráter hidrofóbico proporcionado aos painéis quando do seu uso, o que pode ter levado os componentes dos painéis confeccionados com a inclusão da mesma a uma absorção reduzida de água após 24 horas de imersão. Batista et al. (2007), ao avaliarem as propriedades físicas e mecânicas de chapas aglomeradas para um mesmo teor de adesivo ureia-formaldeído, estudaram a influência da presença e da ausência de parafina sobre essas propriedades. Para a absorção de água, tanto em 2 quanto em 24 horas, os autores encontraram maiores valores para absorção nos referidos intervalos na presença de parafina e sugeriram algum problema com o produto. Segundo Maloney (1996), a aplicação de 1\% de emulsão de parafina, em relação ao peso seco das partículas, aumenta a resistência à absorção de água dos painéis.

A análise de variância indicou efeito significativo da presença de parafina no inchamento em espessura, em 2 horas. Para a variável inchamento em espessura em 24 horas, houve efeito significativo da interação entre os fatores estudados, porcentagem de incorporação de PET x parafina (I x P).

Os resultados apresentados na Tabela 4 mostram que os painéis confeccionados na ausência de parafina apresentaram maior inchamento em espessura após 2 horas de imersão, quando comparados com aqueles confeccionados na presença de parafina para a mesma propriedade. Já para o inchamento em espessura em 24 horas (Tabela 5), esse mesmo comportamento foi observado apenas para os painéis que foram confeccionados na ausência de plástico, ou seja, $0 \%$ de incorporação. Para as demais porcentagens de incorporação de plástico do tipo PET à madeira, essa propriedade foi estatisticamente igual entre si.

TABELA 4: Porcentagem de inchamento em espessura após 2 horas de imersão em água, em função das porcentagens de incorporação de plástico tipo PET à madeira e da presença/ausência de parafina (erro padrão da média).

TABLE 4: Thickness swelling after 2 hours of immersion, as a function of PET percentage combined with wood in paraffin presence/absence.

\begin{tabular}{ccccc}
\hline \multirow{2}{*}{ Parafina } & \multicolumn{3}{c}{ Porcentagem de incorporação de PET } & \multirow{2}{*}{ Médias (\%) } \\
\cline { 2 - 4 } & $0 \%$ & $25 \%$ & $50 \%$ & $10,09(0,70) \mathrm{b}$ \\
Presença & $9,87(1,22)$ & $8,54(1,22)$ & $11,88(1,22)$ & $10,70(0,70) \mathrm{a}$ \\
Ausência & $12,81(1,22)$ & $12,34(1,22)$ & $12,97(1,22)$ & $12,70(0,46)$ \\
\hline Médias (\%) & $11,34(0,86)$ & $10,44(0,86)$ & $12,43(0,86)$ & \\
\hline
\end{tabular}

Médias seguidas de mesma letra minúscula na coluna não diferem entre si, pelo teste t de Student, a 5\% de significância.

TABELA 5: Porcentagem de inchamento em espessura após 24 horas de imersão em água, em função das porcentagens de incorporação de plástico tipo PET à madeira e da presença/ausência de parafina (erro padrão da média).

TABLE 5: Thickness swelling after 24 hours of immersion, as a function of PET percentage combined with wood in paraffin presence/absence.

\begin{tabular}{ccccc}
\hline \multirow{2}{*}{ Parafina } & \multicolumn{3}{c}{ Porcentagem de incorporação de PET } & \multirow{2}{*}{ Médias (\%) } \\
\cline { 2 - 4 } & $0 \%$ & $25 \%$ & $50 \%$ & $14,90(0,58)$ \\
Presença & $13,82(1,01) \mathrm{b}$ & $14,64(1,01) \mathrm{a}$ & $16,11(1,01) \mathrm{a}$ & $16,72(0,58)$ \\
Ausência & $18,60(1,01) \mathrm{a}$ & $15,88(1,01) \mathrm{a}$ & $15,67(1,01) \mathrm{a}$ & 16,010 \\
\hline Médias (\%) & $16,21(0,71)$ & $15,26(0,71)$ & $15,96(0,71)$ & \\
\hline
\end{tabular}

Médias seguidas de mesma letra minúscula na coluna não diferem entre si, pelo teste t de Student, a 5\% de significância.

Ci. Fl., v. 21, n. 1, jan.-mar., 2011 
De acordo com os resultados, o inchamento em espessura, de forma geral, não foi reduzido pela adição de parafina, exceto para os painéis produzidos sem incorporação do PET, em função de uma maior impermeabilização das partículas de madeira que são hidrofilicas. Assim, o emprego da parafina em painéis produzidos apenas com madeira foi fundamental para reduzir o inchamento em espessura, tanto em 2 quanto em 24 horas, após imersão em água. Os valores observados para o inchamento em espessura, tanto após 2 quanto 24h, estão abaixo dos estabelecidos pela norma CS 236-66. A norma estabelece valor máximo para inchamento em espessura de $35 \%$ para painéis de partículas de média densidade.

\section{Propriedades mecânicas}

Os resultados experimentais da análise de variância indicaram que a resistência à compressão paralela nos painéis foi afetada pelos tratamentos, ocorrendo efeito significativo da interação entre os fatores porcentagem de incorporação de PET $\mathrm{x}$ parafina ( $\mathrm{I}$ P), indicando um comportamento diferenciado dessa variável em cada porcentagem de incorporação de PET à madeira, na presença e na ausência de parafina.

Os resultados indicaram, por meio da análise de desdobramento, que os níveis de incorporação de plástico do tipo PET à madeira proporcionaram efeito significativo na presença de parafina na confecção dos painéis.

$\mathrm{Na}$ Figura 3, observa-se que a relação existente entre a porcentagem de incorporação de PET à madeira e a resistência à compressão são relações quadráticas decrescentes, ou seja, com o aumento nos níveis de incorporação de PET à madeira na confecção dos painéis, houve neles decréscimo da resistência à compressão, na presença de parafina. Essa redução da resistência se deve, principalmente, à falta de ancoramento do adesivo no PET, devido a sua baixa permeabilidade.

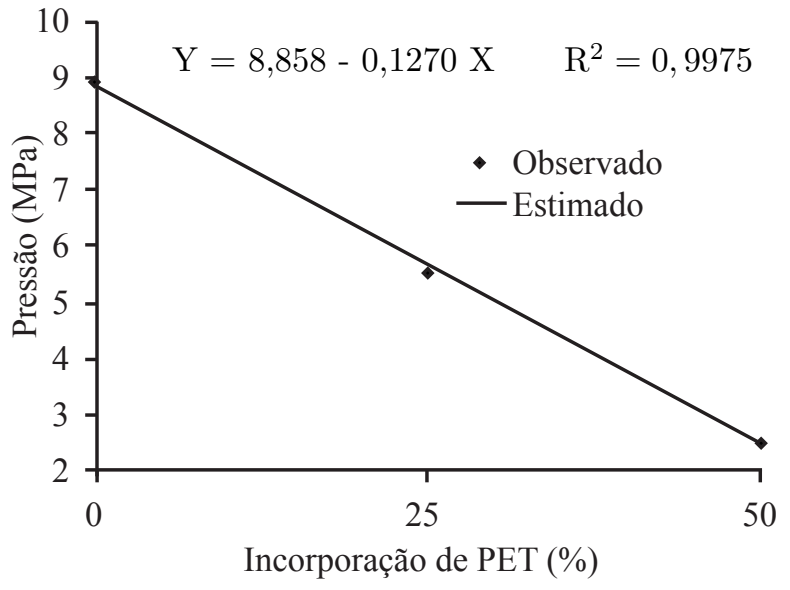

FIGURA 3: Valores médios da variável pressão estimada no teste de compressão, em função das porcentagens de incorporação de PET, na presença de parafina.

FIGURE 3: Average values of the strength estimated in compression test, as a function of PET percentage combined with wood in paraffin presence.

Isso evidencia a necessidade do uso de agentes de acoplamento ou, até mesmo, de tratamentos que possam melhorar a superfície do PET, promovendo uma melhor adesão.

Os valores médios observados para essa propriedade, em função das porcentagens de incorporação de plástico do tipo PET à madeira, na presença e na ausência de parafina, podem ser observados na Tabela 6 .

A maior resistência à compressão paralela foi observada nos painéis produzidos apenas com madeira, na presença de parafina. Provavelmente, a parafina promoveu maior interação dos componentes do painel, alterando fisicamente as superfícies das partículas, acarretando uma redução da higroscopicidade dos painéis, reduzindo a umidade de equilíbrio higroscópico e, conseqüentemente, gerando o aumento da resistência em função da menor umidade. Por outro lado, a adição de

TABELA 6: Resistência a compressão paralela, em função das porcentagens de incorporação de PET, na presença e na ausência de parafina.

TABLE 6: Parallel compression strength, as a function of PET percentage combined with wood in paraffin presence/absence.

\begin{tabular}{ccccc}
\hline \multirow{2}{*}{ Parafina } & \multicolumn{3}{c}{ Porcentagem de incorporação de PET } & \multirow{2}{*}{ Médias (MPa) } \\
\cline { 2 - 4 } & $0 \%$ & $25 \%$ & $50 \%$ & \\
\hline Presença & $8,77(6,97) \mathrm{a}$ & $5,39(6,97) \mathrm{a}$ & $2,54(6,97) \mathrm{a}$ & $5,57(4,02)$ \\
Ausência & $5,90(6,97) \mathrm{b}$ & $5,68(6,97) \mathrm{a}$ & $3,68(6,97) \mathrm{a}$ & $5,09(4,02)$ \\
\hline Médias & $7,33(4,93)$ & $5,54(4,93)$ & $3,11(4,93)$ & \\
\hline
\end{tabular}

Médias seguidas de mesma letra minúscula na coluna não diferem entre si, pelo teste t de Student, a 5\% de significância. 
parafina resulta em uma diminuição da massa das partículas (em relação ao peso seco), o que pode causar, também, redução na resistência mecânica dos painéis.

Os resultados experimentais do teste de flexão estática, em que as variáveis módulo de elasticidade (MOE) e módulo de ruptura (MOR) foram analisadas, indicaram, por meio da análise de variância, comportamento diferenciado entre as variáveis.Houveefeito significativo dasporcentagens de incorporação de plástico em associação à madeira sobre o módulo de elasticidade. Já para a variável módulo de ruptura, não houve efeito significativo de nenhuma das fontes de variação consideradas no modelo, porcentagem de incorporação (I), parafina (P) e interação porcentagem de incorporação de PET x parafina (I x P).

Através da Figura 4, verifica-se uma relação linear decrescente entre as porcentagens de incorporação de PET e a rigidez dos painéis.

Na Tabela 7, observa-se que os valores médios para o módulo de elasticidade em função da adição de parafina aos diferentes tratamentos não apresentam diferença significativa.

Os valores médios para MOR estão apresentados na Tabela 8. Observa-se que não houve efeito significativo dentro das fontes de variação estudadas para essa variável.

Os valores médios para MOE e MOR observados neste trabalho estão abaixo dos valores exigidos pela norma CS 236-66, que estabelece valores mínimos de 2,450 $\mathrm{MPa}$ e 11,2 $\mathrm{MPa}$, respectivamente.
Maciel (2001), estudando painéis de partículas aglomeradas em mistura com plásticos do tipo PET e PS, em diferentes proporções e dois níveis de adesivo (4 e 6\%), não observou diferença significativa, entre os painéis produzidos com e sem plástico, quanto ao módulo de elasticidade.

Apesar dos valores médios para MOE e MOR não apresentarem diferenças estatísticas significativas, deve-se considerar, em relação à composição dos painéis que, neste trabalho, não foi seguido um procedimento comum que é adicionar ao sistema madeira-plástico substâncias

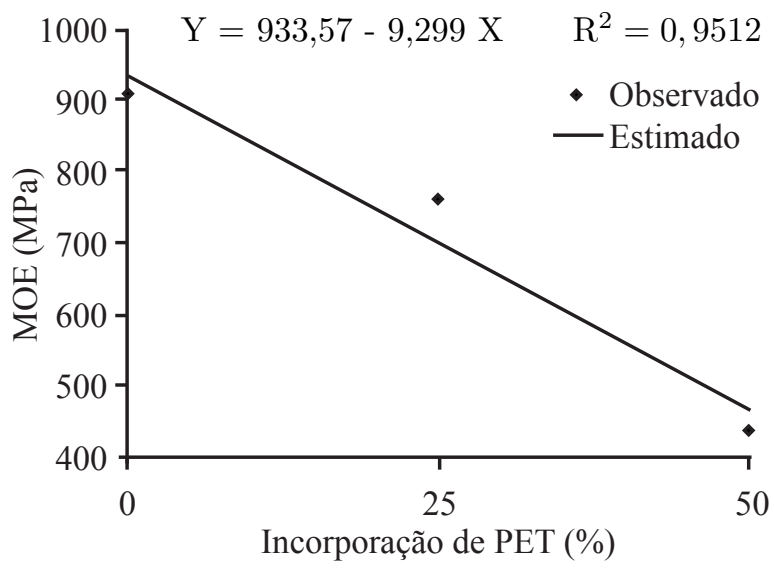

FIGURA 4: Valores médios de MOE em flexão estática, em função das porcentagens de incorporação de PET na confecção dos painéis.

FIGURE 4: MOE as a function of PET percentage combined with wood.

TABELA 7: Valores médios de MOE em flexão estática, em função das porcentagens de incorporação de plástico do tipo PET e da presença/ausência de parafina.

TABLE 7: MOE as a function of PET percentage combined with wood in paraffin presence/absence.

\begin{tabular}{ccccc}
\hline \multirow{2}{*}{ Parafina } & \multicolumn{3}{c}{ Porcentagem de incorporação de PET } & \multirow{2}{*}{ Médias (MPa) } \\
\cline { 2 - 4 } & $0 \%$ & $25 \%$ & $50 \%$ & \\
\hline Presença & $979,59(114,72)$ a & $775,28(114,72)$ a & $426,92(114,72)$ a & $727,27(66,24)$ \\
Ausência & $791,78(114,72)$ a & $719,07(114,72)$ a & $432,56(114,72)$ a & $647,80(66,24)$ \\
\hline Médias (MPa) & $885,69(81,12)$ & $747,17(81,12)$ & $429,74(81,12)$ & \\
\hline
\end{tabular}

Médias seguidas de mesma letra minúscula na coluna não diferem entre si, pelo teste t de Student, a 5\% de significância.

TABELA 8: Valores médios de MOR em flexão estática, em função da incorporação de plástico, na presença ou ausência de parafina.

TABLE 8: MOR as a function of PET percentage combined with wood in paraffin presence/absence.

\begin{tabular}{ccccc}
\hline \multirow{2}{*}{ Parafina } & \multicolumn{3}{c}{ Porcentagem de incorporação de PET } & \multirow{2}{*}{ Médias (MPa) } \\
\cline { 2 - 4 } & $0 \%$ & $25 \%$ & $50 \%$ & \\
\hline Presença & $6,56(1,13)$ & $5,65(1,13)$ & $9,40(1,13)$ & $7,21(0,65) \mathrm{a}$ \\
Ausência & $8,05(1,13)$ & $9,02(1,13)$ & $8,33(1,13)$ & $8,47(0,65) \mathrm{a}$ \\
\hline Médias (MPa) & $7,30(0,80)$ & $7,33(0,8)$ & $8,86(0,8)$ & \\
\hline
\end{tabular}

Médias seguidas de mesma letra minúscula na coluna não diferem entre si, pelo teste t de Student, a 5\% de significância. 
compatibilizantes que possam atuar como elo entre as superfícies desses dois materiais, consequentemente, fortalecendo as linhas de adesão entre os constituintes, gerando produtos de melhor qualidade (SIMONSEN et al., 1997, citados por MACIEL, 2001). Sugere-se, ainda, em relação aos parâmetros de produção dos painéis, que o calor fornecido ao compósito durante a prensagem do material não foi suficiente para fundir, parcialmente, o material plástico agregado às partículas de madeira. Vale salientar que temperaturas de prensagem acima de $250^{\circ} \mathrm{C}$ (temperatura de fusão do PET) inviabilizam economicamente a produção dos painéis, sendo o uso de agentes de acoplamento o método mais econômico para aumentar a resistência dos mesmos.

De acordo com a análise de variância, houve efeito significativo na resistência à ligação interna, em função das porcentagens de incorporação de plástico em associação à madeira na confecção dos painéis.

Pode-se observar, na Figura 5, uma relação linear decrescente entre as porcentagens de incorporação de PET na confecção dos painéis e a resistência à ligação interna.

Os valores médios de resistência à ligação interna, em função das porcentagens de incorporação de PET, na presença e ausência de parafina, estão apresentados na Tabela 9.

Maciel (2001), estudando a inclusão de plástico do tipo PET e PS em painéis de partículas aglomeradas, observou que a resistência à ligação interna dos painéis foi maior naqueles produzidos com menor proporção de plástico, com maior teor de adesivo (6\%) e com adição de solução de poliestireno em tolueno, independente da mistura utilizada, tendo estes resistência superior àqueles produzidos sem a incorporação de plástico. Vale ressaltar que os parâmetros adotados pelo autor diferem dos utilizados neste trabalho, especialmente no que se refere à ausência do uso da parafina e da solução de poliestireno, o que contribui para maior resistência destes painéis. Batista et al. (2007) obtiveram melhores resultados para ligação interna em painéis produzidos com madeira de Pinus, na ausência de parafina, ao estudar o fator presença e ausência de parafina, isoladamente, na confecção dos painéis.

Observa-se, na Tabela 9, que a resistência à ligação interna dos painéis não foi afetada pela adição de parafina, independente da incorporação ou não de plástico (PET).

Apesar de não apresentarem diferenças significativas, os resultados médios observados neste trabalho para resistência à ligação interna em relação à utilização de parafina foram superiores aos valores mínimos estabelecidos pela norma CS-23666, de 0,49MPa. Quanto à incorporação de plástico, verifica-se que os painéis produzidos com $50 \%$ de PET não atingiram o mínimo valor estabelecido pela norma.

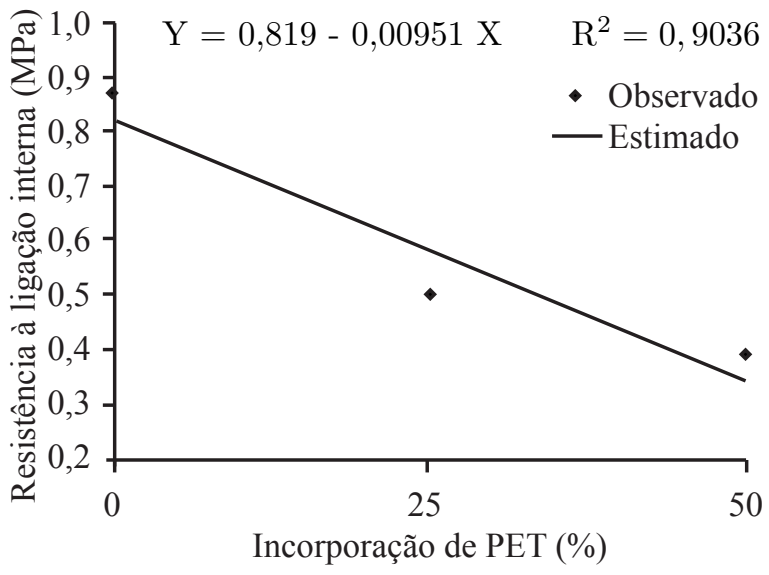

FIGURA 5: Valores médios de resistência à ligação interna em função das porcentagens de incorporação de PET na confecção dos painéis.

FIGURE 5: Internal bond strength as a function of PET percentage combined with wood in panel manufacture.

TABELA 9: Valores médios de ligação interna, em função das porcentagens de incorporação de PET e presença e ausência de parafina.

TABLE 9: Average values of internal bonding as a function of PET percentage combined with wood in paraffin presence/absence.

\begin{tabular}{ccccc}
\hline \multirow{2}{*}{ Parafina } & \multicolumn{3}{c}{ Porcentagem de incorporação de PET } & \multirow{2}{*}{ Médias $(\mathrm{MPa})$} \\
\cline { 2 - 4 } & $0 \%$ & $25 \%$ & $50 \%$ & \\
\hline Presença & $0,85(0,06)$ & $0,49(0,06)$ & $0,33(0,06)$ & $0,55(0,03) \mathrm{a}$ \\
Ausência & $0,84(0,06)$ & $0,47(0,06)$ & $0,42(0,06)$ & $0,58(0,03) \mathrm{a}$ \\
\hline Médias (MPa) & $0,85(0,04)$ & $0,48(0,04)$ & $0,38(0,04)$ & \\
\hline
\end{tabular}

Médias seguidas de mesma letra minúscula na coluna não diferem entre si, pelo teste t de Student, a 5\% de significância. 
Vale ressaltar que os resultados físicos e mecânicos obtidos neste trabalho não devem ser comparados com outros processos de produção, como injeção e extrusão, por exemplo, nos quais ocorre maior homogeneização do material quando da fusão do plástico dentro do equipamento.

\section{CONCLUSÕES E RECOMENDAÇÕES}

O aumento do nível de incorporação de plástico do tipo PET promoveu a redução nos valores das propriedades mecânicas dos painéis aglomerados.

Provavelmente, a redução da resistência mecânica nos painéis, na medida em que se aumentou o nível de incorporação de plástico, foi promovida pela ausência de ancoramento do adesivo ao PET, bem como pela temperatura de prensagem utilizada na confecção dos painéis aglomerados.

De modo geral, a incorporação de PET promoveu, nos painéis confeccionados com ausência de parafina, redução nas porcentagens de absorção de água.

Não foi observado efeito da presença de parafina na absorção de água e inchamento dos painéis à medida que se aumentaram os níveis de incorporação de PET, evidenciando que não existe necessidade de se adicionar parafina em painéis aglomerados confeccionados com adição de plástico do tipo PET.

De modo geral, as propriedades mecânicas não foram afetadas pela adição de parafina, exceto nos painéis confeccionados com ausência de PET.

É viável a utilização do resíduo da madeira de candeia, em associação à madeira de eucalipto, na confecção de painéis aglomerados com adição de PET.

\begin{tabular}{llllr} 
& As & propriedades & \multicolumn{2}{r}{ físico-mecânicas } \\
dos painéis & atenderam & às & exigências \\
mínimas & estabelecidas & nas & normas
\end{tabular} ASTM D 1037 e CS 236-66, exceto para o teste de flexão estática.

Recomenda-se, visando viabilizar a utilização de materiais recicláveis disponíveis, que, nos próximos estudos, sejam adicionadas substâncias compatibilizantes ao processo convencional de produção de painéis aglomerados com adição de plástico do tipo PET, como também a realização de estudos sobre a influência de diferentes temperaturas durante o processo de confecção desses painéis, além do uso de agentes de acoplamento ou até mesmo tratamentos que possam melhorar a superfície do PET, na produção de painéis aglomerados com inclusão desse tipo de plástico.

\section{AGRADECIMENTOS}

À FAPEMIG pelo financiamento desta pesquisa.

\section{REFERÊNCIAS BIBLIOGRÁFICAS}

AMERICAN SOCIETY FOR TESTING AND MATERIALS. ASTM D 1037- 93. Standard test methods for evaluating properties of wood-base fiber and particle panels. Philadelphia, 1995. v. 04. (Annual Book of ASTM Standards).

BATISTA, D. C. et al. Fabricação de aglomerados de três camadas com madeira de Pinus elliottii Engelm. e casca de Eucalyptus pellita Muell. Cerne, Lavras, v. 13, n. 2, p.178-187, mar./abr.2007.

BRANDT, C. W.; FRIDLEY, K. J. Effect of load rate on flexural properties of wood-plastic composites. Wood and Fiber Science, Madison, v.35, n.1, p.135-147, out./nov.2003.

COMMERCIAL STANDARD. CS 236-66. Mat formed wood particleboard. (s.i.), 1968.

FALK, R. H.; VOS, D.; CRAMER, S. M. The comparative performance of woodfiber-plastic and wood- based panels. In: INTERNATIONAL CONFERENCE ON WOODFIBER-PLASTIC COMPOSITES, 5., 1999. Madison. Anais... Proceedings...: Madison, 1999. v. 4, P. 269 - 274. GERMAN STANDARDS COMMITTEE. DIN 68761 - 1971. Specifications for particleboard. Holz, 1971.

HARPER, C. A. Handbook of plastics, elastomers and composites. 3 ed. New York : McGraw-Hill, 1996. 677p.

MACIEL, A. S. Chapas de partículas aglomeradas de madeira de Pinus elliottii ou Eucalyptus grandis, em mistura com poliestireno e polietileno tereftalato. 2001. 115f. Tese (Doutorado em Ciências Florestais) - Universidade Federal de Viçosa, Viçosa, 2001.

MALONEY, T. M. The family of wood composite materials. Forest Products Journal, Madison, v. 46, n. 2, p. 19-26, mar./abr.1996.

VITAL, B.R. Métodos de determinação da densidade da madeira. Viçosa-MG: SIF, 1984. 21p. (Boletim Técnico, 2). 